\title{
Wavelength and Constant Magnetic field Dependence of the Steady-state Photoconductivity of a Bifacial Silicon Solar Cell
}

\section{Diao $A^{*}$ and Sissoko G}

Department of Physics, Faculty of Sciences and Techniques, Semiconductors and Solar Energy Laboratory, Cheikh Anta Diop University, Dakar, Senegal

\begin{abstract}
In this work, a theoretical study on the photoconductivity of a bifacial silicon solar cell under monochromatic illumination and constant magnetic field is presented. Analytical expression of the photoconductivity is established according to base depth, illumination wavelength, junction recombination velocity and magnetic field value. The photoconductivity profile versus wavelength shows two energy absorption peaks corresponding to specific wavelengths. Based on a linear model of the photoconductivity versus junction recombination velocity, in a given interval, we determine an equivalent capacitance which depends on both wavelength and magnetic field.
\end{abstract}

Keywords: Photoconductivity; Wavelength; Recombination velocity; Magnetic field; Capacitance

\section{Introduction}

The photoconductivity is obtained when there is a change in the electrical conductivity of a conductor or a semi-conductor material under electromagnetic radiation [1]. It is linked to the number of free electrons in the conduction band, the number of holes in the valence band, the recombination centers and the impurities doping concentration in a solar cell. Photoconductivity is a characteristic quantity of solar cells allowing access to the density of states and trapping centers in the forbidden band. Therefore, several studies have been conducted on the photoconductivity [2-5]. In particular, in transient state $[6,7]$ a relationship has been founded between the photoconductivity, the average lifetime and the generation rate of photogenerated minority carriers. They deduce from this relationship, the density of states that varies with the absolute temperature. In steady-state, in one hand, without magnetic field, some authors [8-16] determined the photoconductivity according to the temperature, the generation rate, the photocurrent, the incident photons flux (or incident energy) in order to deduce the density of states, and some electrical properties of semiconductors cells. On the other hand, with magnetic field, other researchers $[17,18]$ determined the photoconductivity according to the wavelength, the magnetic field and the incident power. They found the existence of absorption levels which correspond to activation energy and then a negative photoconductivity for certain semiconductor materials.

The purpose of this paper is to determine, by use of the Drüde model and the continuity equation, the photoconductivity according to the wavelength, the applied magnetic field, the base depth and the junction recombination velocity of a silicon solar cell.

\section{Theory}

In Figure 1, we present, a 3D model of an $\mathrm{n}^{+}-\mathrm{p}-\mathrm{p}^{+}$type of a bifacial silicon solar cell $[19,20]$ under magnetic field [21-23].

When the solar cell is irradiated with a monochromatic light, generation and recombination of electron-hole pairs occur in the base. Excited electrons from the valence band, can be trapped in the forbidden band or move directely towards the conduction band by leaving holes behind. The excess of electrons as called excess minority carriers in the p-type base, are subjected to the electrical force $q E$, Lorentz force $q \vec{v} \wedge \vec{B}$ and frictional force $-\frac{m}{\tau_{n}} \vec{v}$ during their diffusion in the crystal lattice; $\mathrm{q}$ and $\tau_{n}$ are respectively the elementary charge and the average lifetime of electrons in the base; $\vec{v}$ is the electron velocity; $\vec{E}$ the electric field of the base; $\vec{B}$ the applied magnetic field.

By applying the basic dynamic principle on electrons, we get eqn. (1):

$m \frac{d \vec{v}}{d t}=q \vec{E}+q \vec{v} \wedge \vec{B}-\frac{m}{\tau_{n}} \vec{v}$

In steady-state, eqn. (1) becomes [24]:

$q \vec{E}+q \vec{v} \wedge \vec{B}-\frac{m}{\tau} \vec{v}=\overrightarrow{0}$

Let's write $\vec{v}$ and $\vec{v}$ in the form:

$$
\begin{aligned}
& \vec{E}=E_{x} \vec{i}+E_{y} \vec{j}+E_{z} \vec{k} \\
& \vec{v}=v_{x} \vec{i}+v_{y} \vec{j}+v_{z} \vec{k} \text { et } \vec{B}=B \vec{j}
\end{aligned}
$$

Substituting eqns. (3) and (4) in eqn. (2), we deduce the velocity components below:

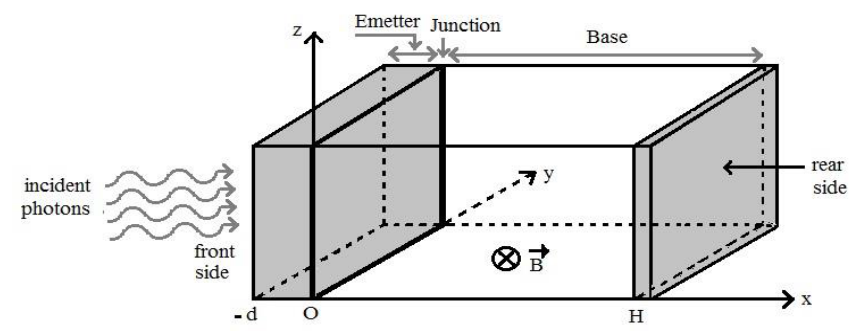

Figure 1: An $n^{+}-p-p^{+}$bifacial silicon solar cell under magnetic field. $d$ and $\mathrm{H}$ are the emitter and base thicknesses respectively.

*Corresponding author: Amadou Diao, Department of Physics, Cheikh Anta Diop University, 5005 Dakar Fann, Senegal, Tel: 00221775515440; E-mail: amadou.diao@ucad.edu.sn, gsissoko@yahoo.com

Received August 03, 2017; Accepted October 03, 2017; Published October 13 2017

Citation: Diao A, Sissoko G (2017) Wavelength and Constant Magnetic field Dependence of the Steady-state Photoconductivity of a Bifacial Silicon Solar Cell. J Material Sci Eng 6: 381. doi: 10.4172/2169-0022.1000381

Copyright: (c) 2017 Diao A, et al. This is an open-access article distributed under the terms of the Creative Commons Attribution License, which permits unrestricted use, distribution, and reproduction in any medium, provided the original author and source are credited. 


$$
\begin{aligned}
& v_{x}=\frac{\mu_{o}}{\left(1+\mu_{o}^{2} B^{2}\right)} E_{x}-\frac{\mu_{o}^{2} B}{\left(1+\mu_{o}^{2} B^{2}\right)} E_{z} \\
& v_{y}=\mu_{o} E_{y} \\
& v_{z}=\frac{\mu_{o}^{2} B}{\left(1+\mu_{o}^{2} B^{2}\right)} E_{x}+\frac{\mu_{o}}{\left(1+\mu_{o}^{2} B^{2}\right)} E_{z}
\end{aligned}
$$

The set of eqns. (5)-(7) can be rewritten in matrix form:

$$
\left(\begin{array}{l}
v_{x} \\
v_{y} \\
v_{z}
\end{array}\right)=\left(\begin{array}{ccc}
\frac{\mu_{o}}{\left(1+\mu_{o}^{2} B^{2}\right)} & 0 & -\frac{\mu_{o}^{2} B}{\left(1+\mu_{o}^{2} B^{2}\right)} \\
0 & \mu_{o} & 0 \\
\frac{\mu_{o}^{2} B}{\left(1+\mu_{o}^{2} B^{2}\right)} & 0 & \frac{\mu_{o}}{\left(1+\mu_{o}^{2} B^{2}\right)}
\end{array}\right)\left(\begin{array}{l}
E_{x} \\
E_{y} \\
E_{z}
\end{array}\right)
$$

Furthermore the current density is the photoconductivity tensor times the electric field as given by eqn. (9):

$$
\vec{J}=(\sigma) \vec{E}
$$

From eqn. (9), we obtain:

$$
(\sigma)=\frac{q \delta_{n} \mu_{o}}{\left(1+\mu_{o}^{2} B^{2}\right)}\left(\begin{array}{ccc}
1 & 0 & -\mu_{o} B \\
0 & \left(1+\mu_{o}^{2} B^{2}\right) & 0 \\
\mu_{o} B & 0 & 1
\end{array}\right)
$$

Where, $\mu_{o}=\frac{q \tau_{n}}{m}$ is the electron mobility in the base without magnetic field; $\delta_{n}$ is the excess minority carriers density in the base.

Now, to simplify our study, we consider only the $\mathrm{x}$ axis and neglect the grain boundaries recombination. Then, the expression of the photoconductivity can be written as:

$$
\sigma_{p h}=\frac{q \mu_{o} \delta_{n}}{\left(1+\mu_{o}^{2} B^{2}\right)}
$$

We see that, the photoconductivity depends on the excess minority carrier's density and magnetic field. In steady-state, excess minority carriers flow is governed by the continuity equation:

$$
D_{n} \frac{\partial^{2} \delta_{n}}{\partial x^{2}}-\frac{\delta_{n}}{\tau_{n}}=-G
$$

With $D_{n}$ being the minority carrier's diffusion coefficient given by:

$$
D_{n}=\frac{D_{o}}{1+\mu_{o}^{2} B^{2}}
$$

Where $D_{o}$ is the minority carriers diffusion coefficient without magnetic field; $G$ is the minority carriers generation rate [25] at position $\mathrm{x}$, whose expression is:

$$
G=\alpha(\lambda) \phi(1-R(\lambda)) e^{-\alpha(\lambda) x}
$$

$\alpha(\lambda)$ and $R(\lambda)$ are respectively the absorption coefficient and the reflection coefficient at wavelength $\lambda ; \varphi$ is the incident photons flux that is supposed relatively constant in this work.

A solution of eqn. (12) is given by:

$$
\delta_{n}=A e^{-\frac{x}{L_{n}}}+C e^{\frac{x}{L_{n}}}-\frac{\alpha(\lambda) L_{n}^{2} \phi(1-R(\lambda)) e^{-\alpha(\lambda) x}}{D_{n}\left(\alpha(\lambda)^{2} L_{n}^{2}-1\right)}
$$

with:

$$
L_{n}=\sqrt{D_{n} \tau_{n}} \text { et }\left(\alpha(\lambda)^{2} L_{n}^{2}-1\right) \neq 0
$$

$L_{n}$ is the minority carrier's diffusion length in the base.
To obtain the complete expression of the minority carriers density, we have to determine coefficients $\mathrm{A}$ and $\mathrm{C}$ by using the following boundary conditions $[26,27]$ :

- At the junction $(\mathrm{x}=0)$,

$\left.D_{n} \cdot \frac{\partial \delta_{n}}{\partial x}\right|_{x=0}=S f .\left.\delta_{n}\right|_{x=0}$

- $\quad$ At the back side $(\mathrm{x}=\mathrm{H})$,

$$
\left.D_{n} \cdot \frac{\partial \delta_{n}}{\partial x}\right|_{x=H}=-\left.S b \cdot \delta_{n}\right|_{x=H}
$$

Where $S f$ and $S b$ are respectively junction recombination velocity and back surface recombination velocity. The recombination velocity $S f$ is the sum of the junction recombination velocity due to the external load that defines the solar cell operating point and the intrinsic junction recombination velocity that materializes an effective recombination velocity of the minority carriers at the emitter-based interface.

To minimize the minority carriers losses at the rear side, we assume the back surface recombination velocity $S b=200 \mathrm{~cm} \cdot \mathrm{s}^{-1}$.

\section{Results and Discussion}

In Figure 2, the photoconductivity is represented according to the base depth for different wavelengths. These curves show that photoconductivity decreases with the base depth. In the vicinity of the junction, at a given operating point of the solar cell, the photoconductivity is maximum because of the important number of photogenerated minority carriers. This can be explained on the one hand, by a high absorption of incident photon in the vicinity of the junction and on the other hand by the decrease of the base absorption due to the Beer-Lambert law and possible recombination of minority carriers in the bulk. We note that for the three wavelengths $\lambda=0.3 \mu \mathrm{m}$, $\lambda=0.5 \mu \mathrm{m}$ and $\lambda=0.7 \mu \mathrm{m}$, the photoconductivity increases in the vicinity of the junction since absorption at the surface is more important than for the bulk. For the wavelength $\lambda=0.9 \mu \mathrm{m}$, the photoconductivity decreases if compared to that obtained with wavelength $0.3 \mu \mathrm{m}$ in the vicinity of the junction but in the bulk of the base, the absorption of the infrared wavelengths can be more important. Therefore, there is a specific wavelength which corresponds to a maximum photoconductivity of the solar cell.

Figure 3 presents the photoconductivity according to the

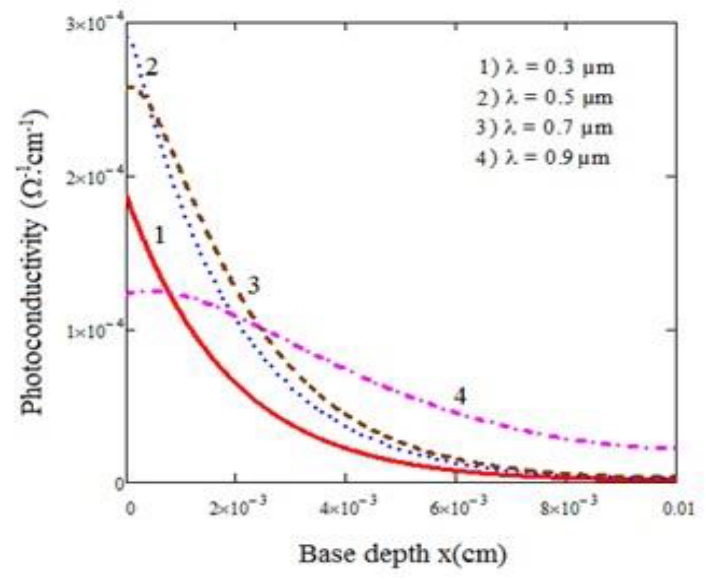

Figure 2: Photoconductivity versus base depth for different wavelengths. 


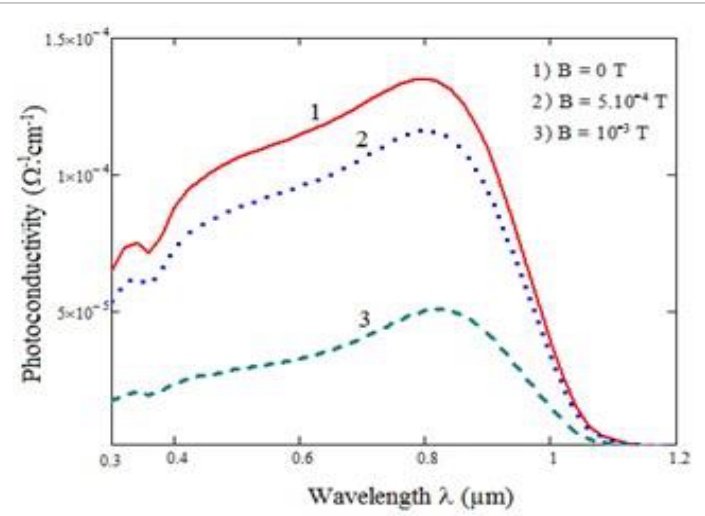

Figure 3: Photoconductivity versus wavelength for different magnetic field values.

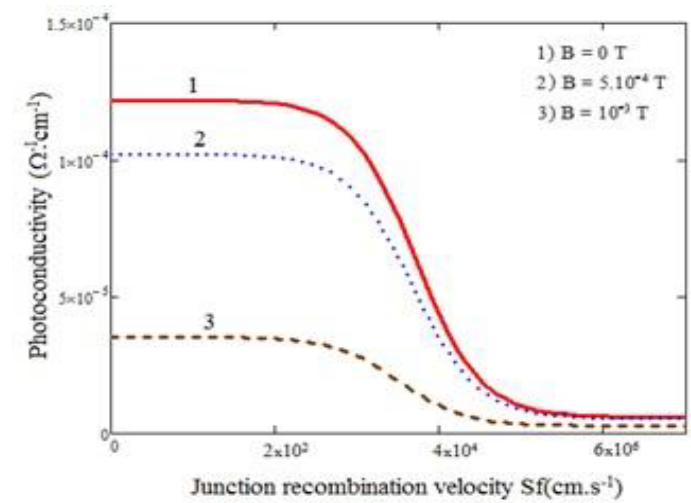

Figure 4: photoconductivity versus junction recombination velocity for different magnetic field values.

wavelength for different magnetic field values. We note the same appearance for all these three curves of photoconductivity according to the wavelength. For a given curve, the maximum of photoconductivity is localized within the visible domain of wavelengths. There are two peaks of photoconductivity corresponding to two specific wavelengths. The first peak corresponds to the wavelength $\lambda=0.34 \mu \mathrm{m}$ for the three curves while the second, for curves 1 and 2, corresponds to $\lambda=0.8 \mu \mathrm{m}$. For curve 3 , the second peak corresponds to the wavelength $\lambda=0.82 \mu \mathrm{m}$. The corresponding photons energies of wavelengths $0.34 \mu \mathrm{m}, 0.8 \mu \mathrm{m}$ and $0.82 \mu \mathrm{m}$, are respectively $3.7 \mathrm{eV}, 1.6 \mathrm{eV}$ and $1.5 \mathrm{eV}$. The first energy $(3.7 \mathrm{eV})$ is attributed to an activation energy of the recombination centers within the forbidden band gap and the second one $(1.6 \mathrm{eV}$ or $1.5 \mathrm{eV}$ ) to an inter-band absorption i.e valence band to conduction band since characterized by high absorption coefficient. Beyond the second peak on Figure 3, the base of the solar cell becomes more and more transparent to the wavelengths because of a feeble absorption coefficient of the material so that the photoconductivity also decreases.

Figure 4 shows that the photoconductivity decreases with the increase of the junction recombination velocity since there is an increase of the excess minority carriers through the junction. For a given magnetic field, we can see, three regions:

- The first, in the range $\left(0 \mathrm{~cm} \cdot \mathrm{s}^{-1} ; 2.102 \mathrm{~cm} . \mathrm{s}^{-1}\right)$ where the solar cell operates near open circuit with a quite constant photoconductivity. The excess minority carriers are stored near the junction since they haven't enough kinetic energy to cross this junction;

\begin{tabular}{|l|c|c|c|}
\hline $\mathrm{B}(\mathrm{T})$ & 0 & $5.10^{-4}$ & $10^{-3}$ \\
\hline$\sigma_{\text {pho }}\left(10^{-4} \Omega^{-1} \cdot \mathrm{cm}^{-1}\right)$ & 1.21 & 1.01 & 0.35 \\
\hline $\mathrm{C}\left(\mathrm{pF} . \mathrm{cm}^{-2}\right)$ & 229 & 190 & 65 \\
\hline
\end{tabular}

Table 1: A few values of $\mathrm{C}$ and $\sigma_{\text {pho }}$.

- The second region, in the range $\left(2.102 \mathrm{~cm} \cdot \mathrm{s}^{-1} ; 5.105 \mathrm{~cm} \cdot \mathrm{s}^{-1}\right)$, corresponds to a variable operating point of the solar cell and where the photoconductivity decreases with the junction recombination velocity. The photogenerated minority carriers have gradually more and more kinetic energy that permits them to cross the junction while the load decreases. That situation leads to the decrease of the minority carriers storage near the junction;

- The third region where the solar cell is operating in short circuit in the range $\left(5.105 \mathrm{~cm} \cdot \mathrm{s}^{-1} ; 7.107 \mathrm{~cm} . \mathrm{s}^{-1}\right)$. The photocreated minority carriers have enough kinetic energy to cross the junction. There is no more significant storage of the minority carriers and the photoconductivity remaining constant is lower than that corresponding to the open circuit situation.

Figure 4 also shows that magnetic field causes a decrease in the magnitude of the photoconductivity: it is the magnetophotoconductivity effect. In fact, with a magnetic field, there is a magnetic force that acts on the minority carriers by deviating them from their initial trajectories and deflecting them towards the surface or the dandling states.

In order to determine the capacitance of the solar cell, when considering the space charge region as a plan capacitor in the range $\left(2.102 \mathrm{~cm} . \mathrm{s}^{-1} ; 5.105 \mathrm{~cm} . \mathrm{s}^{-1}\right)$, we can apply the linear model of this part of the photoconductivity behavior. We obtain a relationship between the photoconductivity and the junction recombination velocity in the form:

$$
\sigma_{p h}=-C . S f+\sigma_{p h o}
$$

Where $\sigma_{p h o}$ corresponds to the photoconductivity in open circuit; $(-\mathrm{C})$ is the slope of the linear part that's concerned here and $\mathrm{C}$ being the capacitance.

Table 1, presents the values of $\mathrm{C}$ and $\sigma_{\text {pho }}$ for different magnetic field values. Both capacitance and photoconductivity in open circuit decrease with the magnetic field. The decrease of the capacitance and the photoconductivity is linked to the lake of minority carriers in the space charge region since there is no significant storage of these photogenerated carriers.

\section{Conclusion}

A theoretical study on the photoconductivity of a bifacial silicon solar cell has been done. From the photoconductivity profiles according to the wavelength and the base depth, a maximum value of photoconductivity can be either obtained with a specific wavelength or in the vicinity of the junction. These two cases above may help in optoelectronic applications and magnetic field detection, to use an optimum base thickness and to choose judiciously a wavelength that gives higher photoconductivity. By means of the linear model of the photoconductivity versus the junction recombination velocity, an equivalent capacitance has been determined when considering the space charge region of the solar cell modelled as a plan capacitor.

\section{References}

1. Sinton RA, Cuevas A, Stuckings M (1996) Quasi-steady-state photoconductance, 
Citation: Diao A, Sissoko G (2017) Wavelength and Constant Magnetic field Dependence of the Steady-state Photoconductivity of a Bifacial Silicon Solar Cell. J Material Sci Eng 6: 381. doi: 10.4172/2169-0022.1000381

a new method for solar cell material and device characterization. Photovoltaic Specialists Conference, 1996., Conference Record of the Twenty Fifth IEEE pp: $457-460$

2. Bube RH (1978) Photoconductivity of solids. RE Krieger Pub. Co.

3. Bube RH (1974) Electronic Proporties of Crystalline Solids. Academic Press, New York.

4. Joshi NV (Ed.) (1990) Photoconductivity: art: science \& technology (Vol. 25), CRC Press.

5. Orton JW (2004) The story of semiconductors. Oxford University Press.

6. Adriaenssens GJ, Baranovskii SD, Fuhs W, Jansen J, Öktü Ö (1995) Photoconductivity response time in amorphous semiconductors. Physical Review B 51(15).

7. Belgacem H, Merazga A (2008) Determination of the density of localized states in semiconductors from the pre-recombination transient photoconductivity. Solid-State Electronics 52: 73-77.

8. Sinton RA, Cuevas A (1996) Contactless determination of current-voltage characteristics and minority-carrier lifetimes in semiconductors from quasisteady-state photoconductance data. Applied Physics Letters 69: 2510-2512.

9. Feng $Q$, Jinzhong $X$, Jincheng $K$, Lianjie $Y$, Lingde $K$, et al. (2011) Dark conductivity and photoconductivity of amorphous $\mathrm{Hg} 0.78 \mathrm{Cd} 0.22 \mathrm{Te}$ thin films. Journal of Semiconductors 32: 033004.

10. Qamhieh N, Adriaenssens GJ (2001) Steady-state photoconductivity in amorphous germanium selenide films. Journal of non-crystalline solids 292 : 80-87.

11. Pal RK, Ji K, Agnihotri AK, Singh CP, Yadav S, et al. (2009) Temperature and Intensity Dependence of Photoconductivity in a-Se70Te26Zn4: Determination of Defect Centres. Chalcogenide Letters 6: 29-34.

12. Reis FT, Chambouleyron I (2002) Temperature dependence of the photoconductivity of arsenic-doped hydrogenated amorphous germanium thin films. Journal of non-crystalline solids 299: 179-184.

13. Sharmin M, Choudhury S, Akhtar N, Begum T (2012) Optical and transport properties of p-type GaAs. Journal of Bangladesh Academy of Sciences 36: 97-107.

14. Schmidt JA, Longeaud C, Kleider JP (2005) Light-intensity dependence of the steady-state photoconductivity used to estimate the density of states in the gap of intrinsic semiconductors. Thin solid films 493: 319-324.

15. Sharmin M, Choudhury S, Begum T (2015) Electrical Optical and Structural Properties of p-type Silicon. Dhaka University Journal of Science 63: 37-41.

16. Vul BM, Vavilov VS, Plotnikov AF, Sokolova AA, Chapnin VA (1966) Optica absorption, photoconductivity and pn junctions in cadmium telluride. Revue de Physique Appliquée 1: 217-221.

17. Omar MS, Abbas TA (2010) Magneto-Optical properties of GaP single crystal. Iranian Journal of Physics Research 9: 358-358.

18. Heisel W, Bohm W, PrettI W (1981) Negative FIR-photoconductivity in n-GaAs. International Journal of Infrared and Millimeter Waves 2: 829-837.

19. Meier DL, Hwang JM, Campbell RB (1988) The effect of doping density and injection level on minority-carrier lifetime as applied to bifacial dendritic web silicon solar cells. IEEE Transactions on Electron devices 35: 70-79.

20. Hübner A, Aberle AG, Hezel R (1997) 20\% efficient bifacial silicon solar cells In Proceedings of the 14th European Photovoltaic Solar Energy Conference pp: 92-95.

21. Madougou S, Made F, Boukary MS, Sissoko G (2007) IV Characteristics for Bifacial Silicon Solar Cell under a Magnetic Field. In Advanced Materials Research 18: 303-312. Trans Tech Publications.

22. Thiam N, Diao A, Ndiaye M, Dieng A, Thiam A, et al. (2012) Electric equivalent models of intrinsic recombination velocities of a bifacial silicon solar cell under frequency modulation and magnetic field effect. Research Journal of Applied Sciences, Engineering and Technology 4: 4646-4655.

23. Madougou S, Made F, Boukary MS, Sissoko G (2007) Recombination Parameters Determination by Using Internal Quantum Efficiency (IQE) Data Of Bifacial Silicon Solar Cells. In Advanced Materials Research 18: 313-324. Trans Tech Publications.

24. Kittel C (1972) Introduction à la physique de l'état solide: maîtrise de physique.
25. Van Roosbroeck W (1950) Bell Syst Tech J 29: 560-607.

26. Sissoko G, Nanema E, Corréa A, Biteye PM, Adj M, et al. (1998) Silicon solar cell recombination parameters determination using the illuminated IV characteristic. In Proceedings of World Renewable Energy Congress, Florence-Italy, pp: 1848-1851.

27. Sissoko G, Sivoththanam S, Rodot M, Mialhe P (1992) Constant IlluminationInduced Open Circuit Voltage Decay (CIOCVD) method, as applied to high efficiency Si Solar cells for bulk and back surface characterization. In 11th European Photovoltaic Solar Energy Conference and Exhibition, poster B 1: $12-16$. 